\title{
Limited Impact of Anti-PD-1/PD-L1 Monotherapy for Hepatocellular Carcinoma
}

\author{
Masatoshi Kudo \\ Department of Gastroenterology and Hepatology, Kindai University Faculty of Medicine, \\ Osaka-Sayama, Japan
}

Prof. M. Kudo

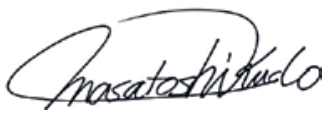

Editor Liver Cancer

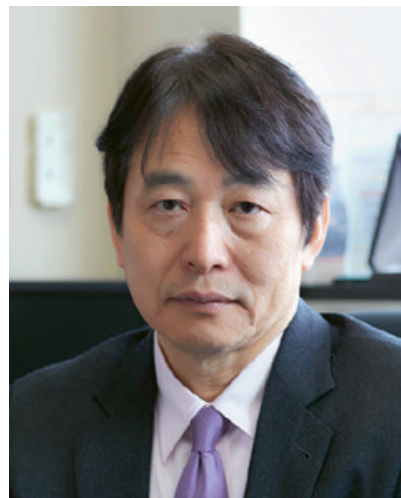

\section{Keywords}

Immune checkpoint inhibitors - Atezolizumab - Bevacizumab - Combination immunotherapy

\section{Introduction}

Immune checkpoint inhibitor (ICI) monotherapy yields lower response rates and higher progressive disease (PD) rates than the recently developed combination immunotherapy regimen [1, 2]. Addition of an anti-vascular endothelial growth factor (VEGF) antibody/ tyrosine kinase inhibitor (TKI) to an anti-PD-1/PD-L1 antibody shifts the immunosuppressive tumor microenvironment (TME) to an immune-permissive microenvironment, which may contribute to the positive results of combination therapy. A second contributing factor is that, although ICI monotherapy is ineffective against hepatocellular carcinoma (HCC) with WNT/ $\beta$-catenin activating mutations, addition of an anti-VEGF antibody or TKI (which are effective as monotherapy in patients with WNT/ $\beta$-catenin mutations) should yield a lower PD rate, and consequently a higher response rate. A third factor is that combination therapy might produce synergistic effects by reversing the immunosuppressive TME even in patients with WNT/ $\beta$-catenin signaling activation or reduced immunogenicity of another origin. 


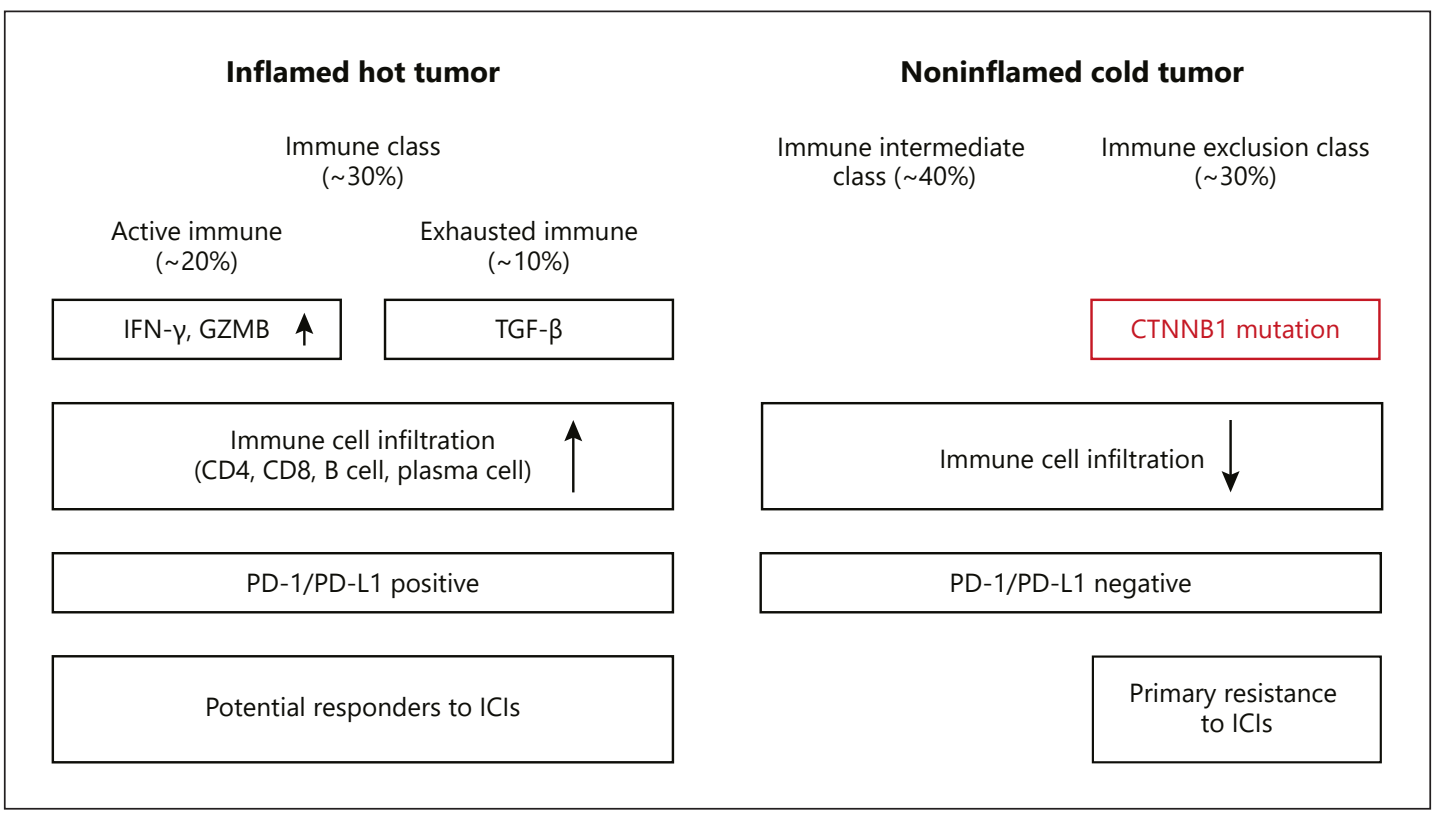

Fig. 1. Immune subclass in hepatocellular carcinoma [artwork based on 8, 9]. ICIs, immune checkpoint inhibitors.

Two ICIs are approved by the United States Food and Drug Administration (FDA) for HCC: nivolumab as second-line therapy following progression on sorafenib (based on the results of the phase I/II CheckMate 040 study) [3], and pembrolizumab as second-line therapy (based on the phase I/II KEYNOTE-224 study) [4]. However, the confirmatory phase III CheckMate 459 study [5] (nivolumab vs. sorafenib) and the second-line phase III KEYNOTE-240 study [6] (pembrolizumab vs. placebo) both failed; therefore, approval of nivolumab and pembrolizumab was withheld in most countries.

The results from the first interim analysis of a study published in the New England Journal of Medicine show that combination therapy with atezolizumab plus bevacizumab is markedly superior to sorafenib as the current standard for all endpoints (overall survival, progressionfree survival, objective response rate, quality of life, and adverse events) [7]. The regimen was already approved by the FDA followed by approval in many countries, including Japan. The success of first-line combination therapy with atezolizumab and bevacizumab is thus surprising considering the failure of first- and second-line monotherapy with ICIs. This editorial explores the limitations of ICIs as monotherapy.

\section{Difference between Anti-PD-1/PD-L1 Monotherapy and Combination Therapy with Anti-PD-1/PD-L1 Antibody plus Anti-VEGF Antibody or TKIs}

To understand the difference between anti-PD-1/PD-L1 antibody as monotherapy versus its use in combination with an anti-VEGF antibody or TKIs, it is first important to examine the immune class of HCC as described by Sia, Llovet and colleagues [8, 9] (Fig. 1). According to Sia, Llovet and colleagues, $20-30 \%$ of all HCC patients have an "immune hot" tumor with lymphocytic infiltration (Fig. 1). Tumors in the immune hot subclass are heavily infiltrated with interferon gamma and granzymes or immune cells. Also, PD-L1 expression is present on the tumor cells. Therefore, those tumors should respond to ICIs. Patients in this subclass have either a type I or type IV TME, as described by Teng et al. [10] (Fig. 2). Patients in the active 


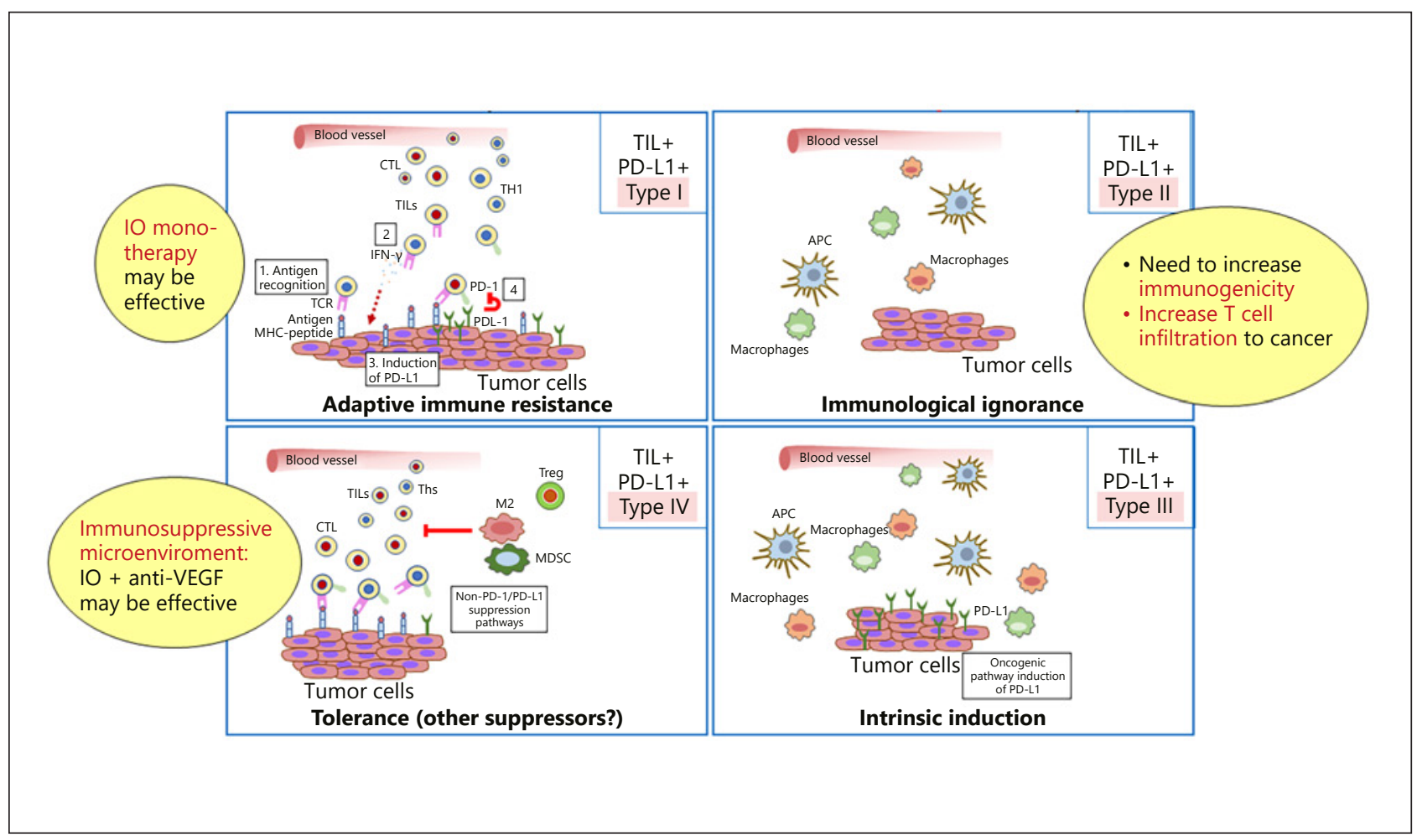

Fig. 2. Cancer is classified into four types depending on immune microenvironment (TIL: CD8+ cell and PDL1 expression) (type I-IV) [modified from 10]. VEGF, vascular endothelial growth factor.

immune class (20\%) who have a Teng type I TME are responsive to ICI monotherapy. Patients in the "immune-exhausted" class (10\%) have suppressed T cell activity due to the presence of various immunosuppressive factors in the TME; this results in a Teng type IV TME, which responds poorly to ICI monotherapy. To summarize, anti-PD-1/PD-L1 antibody monotherapy is effective against tumors in the active immune subclass, whereas it is unlikely to be effective against tumors in the immune-exhausted subclass with an immunosuppressive TME. Addition of an anti-VEGF antibody or TKI therapy should reverse the immunosuppressive TME and allow the anti-PD-1/PD-L1 antibody to activate CD8-positive cells (Fig. 3).

WNT $/ \beta$-catenin mutations that activate $\beta$-catenin are found in $20-30 \%$ of patients with HCC (Fig. 1). Sia, Llovet and colleagues $[8,9]$ classify HCC with WNT/ $\beta$-catenin mutations in the immune exclusion class and propose that this class is unresponsive to ICIs (i.e., exhibits primary resistance).

\section{Differences in the Mechanisms Underlying Monotherapy with Anti-PD-1/PD-L1 Antibody and Combination Therapy with anti-PD-1/PD-L1 Antibody plus Anti- VEGF Antibody or TKIs}

As shown in Table 1, anti-PD-1/PD-L1 monotherapy (1) specifically inhibits the PD-1/ PD-L1 pathway and thus cannot reverse the effects of an immunosuppressive TME or $\beta$-catenin mutation. By contrast, combination therapy with an anti-PD-1/PD-L1 antibody plus an anti-VEGF antibody (e.g., bevacizumab) or TKIs exerts compound effects: (2) it has direct antitumor effects by inhibiting VEGF-A signal transduction produced by anti-VEGF antibody/ 


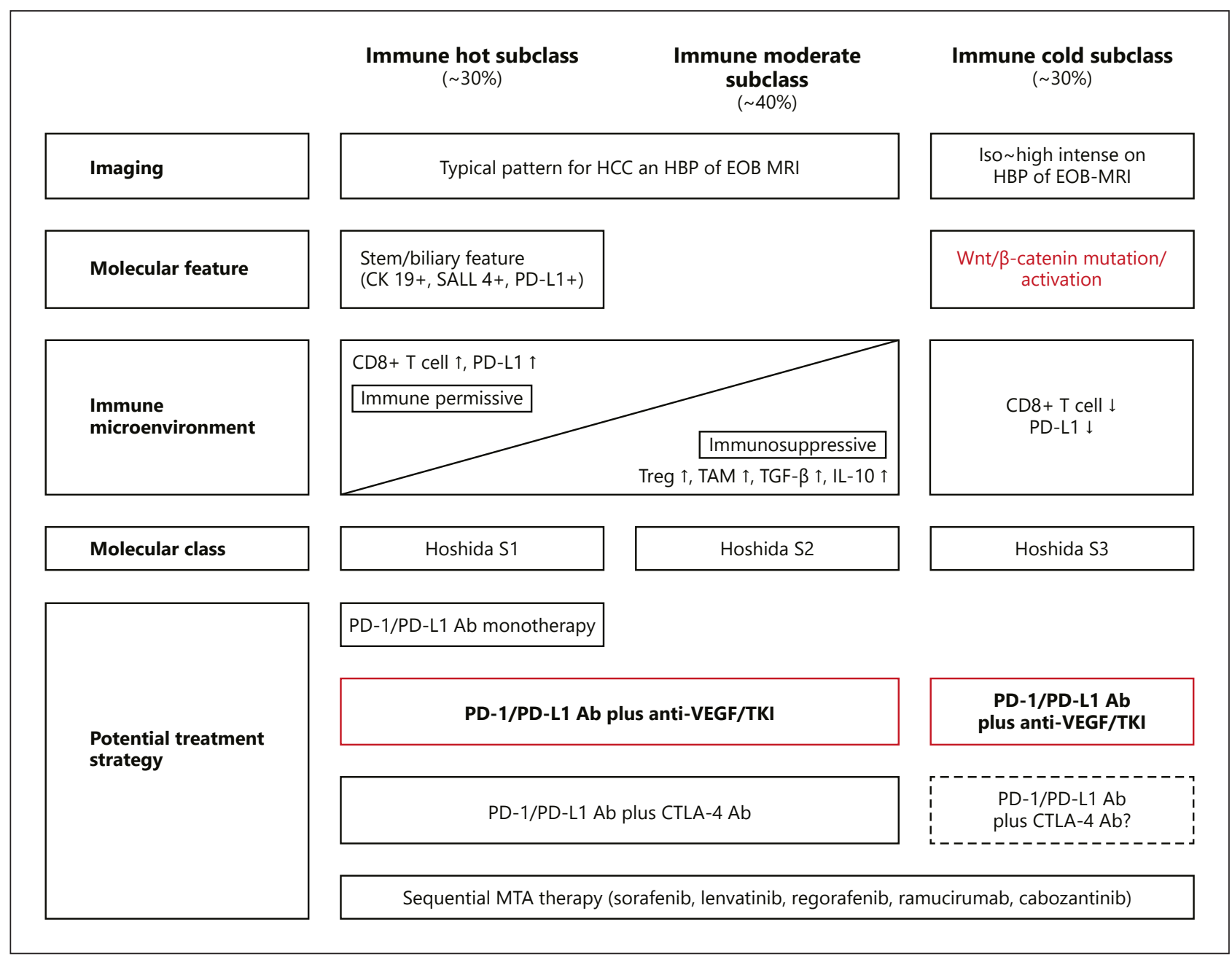

Fig. 3. Immunological classification and possible treatment strategy. Produced based on previous studies [8, 9, 23-26]. HCC, hepatocellular carcinoma; MTA, molecular targeted agents; TKI, tyrosine kinase inhibitor; Treg, regulatory T cells; HBP, hepatobiliary phase.

TKIs even in WNT/ $\beta$-catenin mutated HCC; (3) it increases release of tumor antigens by inducing necrosis in HCC; (4) it activates maturation of dendritic cells and improves recognition and presentation of tumor antigens through the anti-VEGF antibody/TKIs (5), which increases the ability to activate CD8-positive cells in the priming phase; (6) it promotes tumor infiltration by CD8-positive cells through the effect of the anti-VEGF antibody/TKIs on normalizing tumor vasculature; and (7) it reverses the immunosuppressive TME through anti-VEGF effects. Combination therapy with an anti-VEGF antibody or TKIs exerts synergistic effects by simultaneously inhibiting the PD-1/PD-L1 pathway and stimulating immunity at each step of the cancer immunity cycle [11] (Fig. 4; Table 1). Combination therapy activates antitumor immunity at almost every step of the cancer immunity cycle by (1) promoting release of tumor antigens, (2) increasing the antigen-presenting ability of dendritic cells, (3) activating antigen-specific $\mathrm{T}$ cells by improving the priming potential in lymph nodes, (4) promoting tumor infiltration by CD8-positive cells to induce T cell activation, and (5) attacking cancer cells by effectively blocking the PD-1/PD-L1 pathway with improvement of the immunosuppressive TME. 


\begin{tabular}{ll|l}
\cline { 2 - 2 } Liver Cancer 2020;9:629-639 \\
\cline { 2 - 2 } & \multicolumn{2}{l}{$\begin{array}{l}\text { DOl: 10.1159/000512170 } 2020 \text { The Author(s). Published by S. Karger AG, Basel } \\
\text { www.karger.com/lic }\end{array}$} \\
\cline { 2 - 3 }
\end{tabular}

Kudo: Anti-PD-1/PD-L1 Monotherapy for HCC

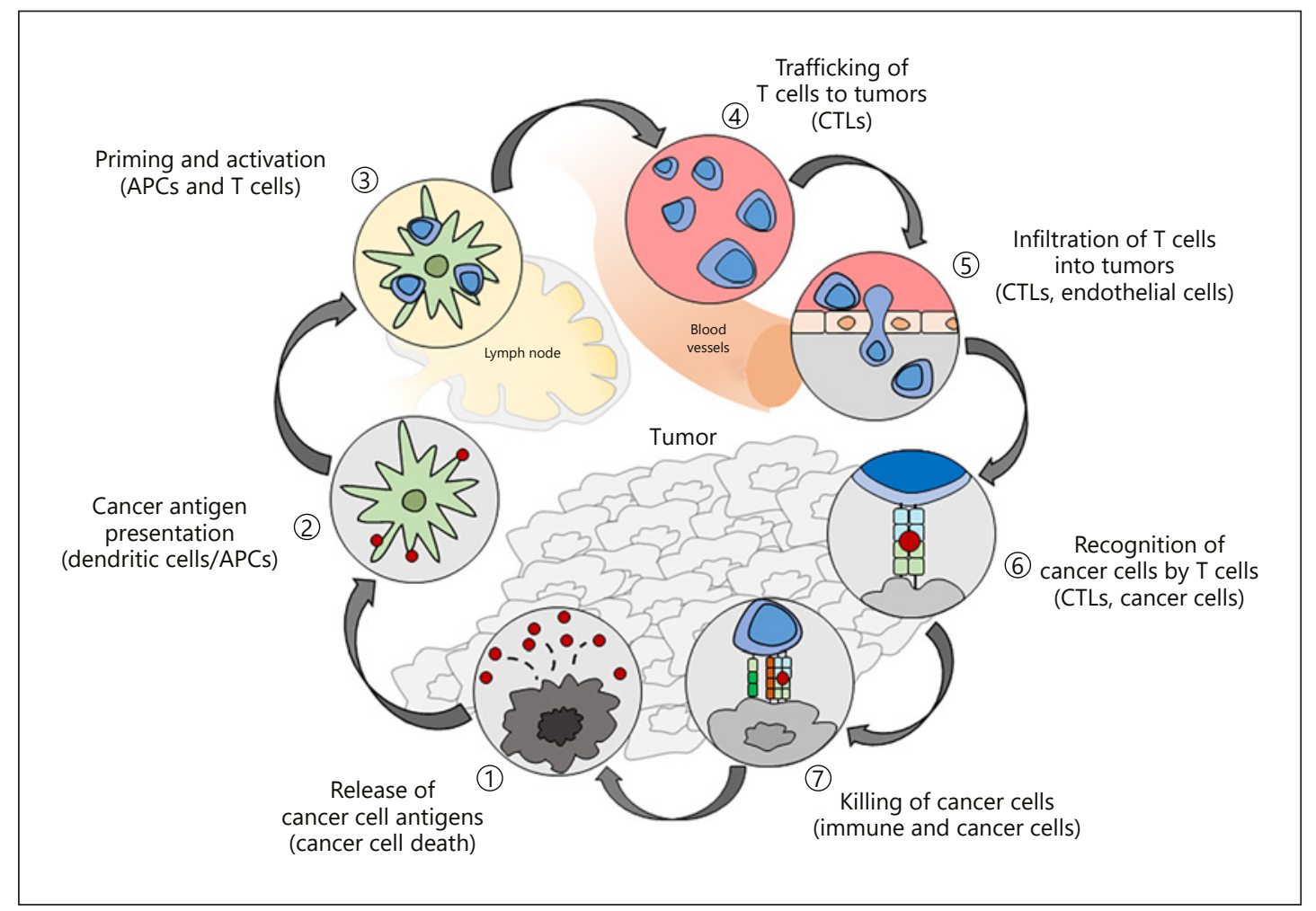

Fig. 4. The cancer-immunity cycle [modified from 11].

Table 1. Synergistic effects of anti-PD-1/PD-L1 antibody + anti-VEGF antibody/TKI combination therapy

\begin{tabular}{|c|c|c|}
\hline & $\begin{array}{l}\text { Anti-PD-1/PD-L1 antibody } \\
\text { monotherapy }\end{array}$ & Anti-PD-1/PD-L1 antibody + anti-VEGF/TKI combination therapy \\
\hline \multirow{7}{*}{$\begin{array}{l}\text { Mechanism } \\
\text { of action }\end{array}$} & 1. PD-1/PD-L1 pathway inhibition & 1. PD-1/PD-L1 pathway inhibition \\
\hline & \multirow{6}{*}{ Effects of anti-VEGF antibody/TKI } & $\begin{array}{l}\text { 2. Direct antitumor effect by inhibition of signal transduction } \\
\text { including WNT/ } \beta \text {-catenin mutated HCC }\end{array}$ \\
\hline & & 3. Increased release of tumor antigens through necrosis of HCC \\
\hline & & $\begin{array}{l}\text { 4. Improved ability to recognize and present tumor antigens through } \\
\text { maturation of dendritic cells }\end{array}$ \\
\hline & & 5. Improved ability to activate CD8-positive cells in the priming phase \\
\hline & & $\begin{array}{l}\text { 6. Increased tumor infiltration by CD8-positive cells through } \\
\text { normalization of tumor vasculature }\end{array}$ \\
\hline & & $\begin{array}{l}\text { 7. Change the immunosuppressive tumor microenvironment to } \\
\text { immune-permissive tumor microenvironment }\end{array}$ \\
\hline
\end{tabular}

HCC, hepatocellular carcinoma; TKI, tyrosine kinase inhibitor; VEGF, vascular endothelial growth factor. 


\begin{tabular}{ll|l} 
Liver Cancer & \multicolumn{2}{l}{ Liver Cancer 2020;9:629-639 } \\
\cline { 2 - 3 } & DOI: 10.1159/000512170 & $\begin{array}{l}\text { @ } 2020 \text { The Author(s). Published by S. Karger AG, Basel } \\
\text { www.karger.com/lic }\end{array}$ \\
\cline { 2 - 3 }
\end{tabular}

Kudo: Anti-PD-1/PD-L1 Monotherapy for HCC

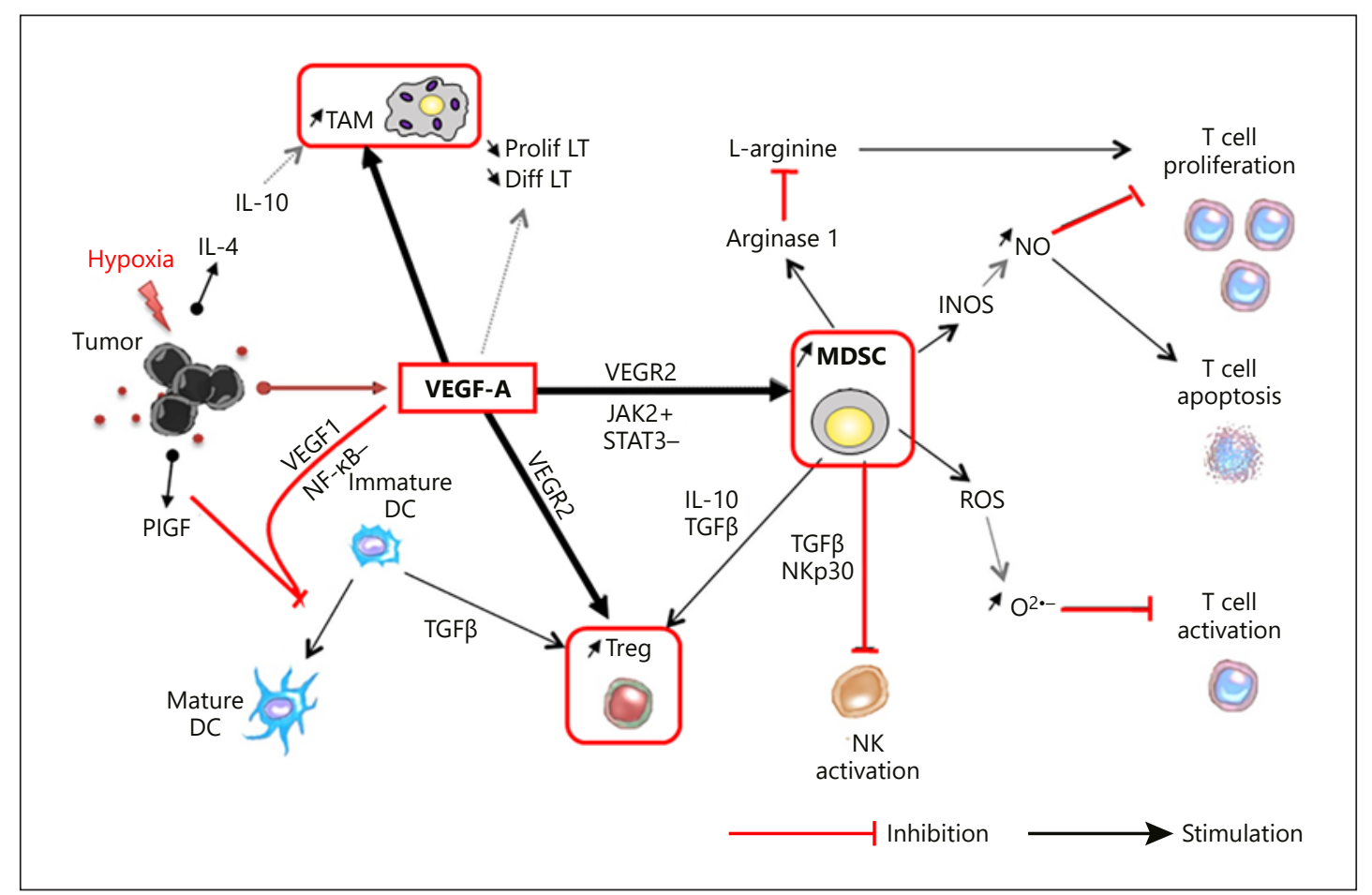

Fig. 5. Mechanism of immunosuppressive tumor microenvironment produced by VEGF [modified from 12]. DC, dendritic cell; MDSC, myeloid-derived suppressor cell; TAM, tumor-associated macrophage; Treg, regulatory T cell; VEGF, vascular endothelial growth factor.

\section{Reversal of the Immunosuppressive TME by Anti-VEGF Antibody}

Release of VEGF-A by hypoxic tumors induces immunosuppressive cells such as tumorassociated macrophages, regulatory $\mathrm{T}$ cells, and myeloid-derived suppressor cells [12] (Fig. 5). Release of immunosuppressive cytokines such as IL-10 and TGF- $\beta$ further shifts the TME toward an immunosuppressive phenotype, which inhibits maturation of dendritic cells and activation and proliferation of T cells (Fig. 5). However, treatment with an anti-VEGF antibody reverses these immunosuppressive effects and promotes $\mathrm{T}$ cell activation and dendritic cell maturation [13] (Fig. 6). Therefore, even in type IV tumors that are infiltrated by CD8-positive cells but in which cell activation does not take place due to the immunosuppressive TME (Fig. 2), an anti-VEGF antibody restores immune activation of CD8-positive cells.

\section{Effects of $\mathrm{ICI}$ Monotherapy and Combination Therapy in Patients with WNT/ $\beta$-Catenin Mutations}

Tumors with a Teng type II TME (Fig. 2) include not only those with inherently low immunogenicity, but also those with WNT/ $\beta$-catenin mutations, as described by Sia, Llovet and colleagues $[8,9]$; thus, they are not infiltrated by CD8-positive cells. Therefore, anti-VEGF antibody or TKI therapy may work in such Teng type II tumors $[14,15]$ without immune cell infiltration by increasing immunogenicity through direct antitumor and necrotic effects on HCC. Harding et al. [16] demonstrated this effect in patients with HCC; they found that all 


\begin{tabular}{ll|l} 
Liver Cancer & \multicolumn{2}{l}{ Liver Cancer 2020;9:629-639 } \\
\cline { 2 - 3 } DOI: 10.1159/000512170 & $\begin{array}{l}\text { O 2020 The Author(s). Published by S. Karger AG, Basel } \\
\text { www.karger.com/lic }\end{array}$ \\
\cline { 2 - 2 }
\end{tabular}

Kudo: Anti-PD-1/PD-L1 Monotherapy for HCC

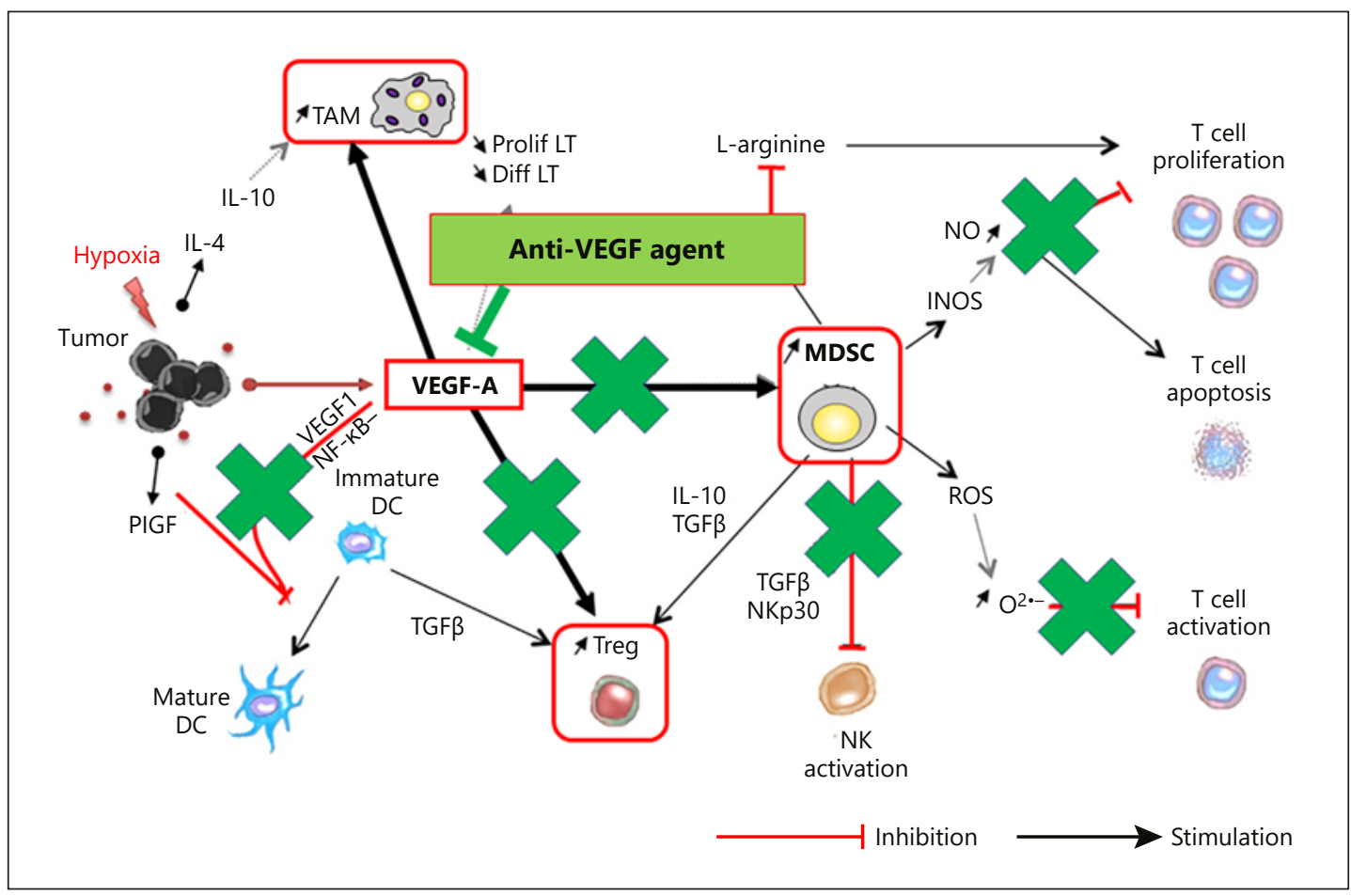

Fig. 6. Improvement of tumor microenvironment from immunosuppressive to immune-permissive by antiVEGF antibody [modified from 12]. DC, dendritic cell; MDSC, myeloid-derived suppressor cell; TAM, tumorassociated macrophage; Treg, regulatory T cell; VEGF, vascular endothelial growth factor.

Table 2. Efficacy of ICIs in patients with advanced HCC $(n=27)$ [cited from 16]

\begin{tabular}{|c|c|c|c|c|}
\hline & $\begin{array}{l}\text { All patients } \\
(n=27)\end{array}$ & $\begin{array}{l}\text { WNT/ } \beta \text {-catenin } \\
\text { mutation/activation }(+) \\
(n=10)\end{array}$ & $\begin{array}{l}\text { WNT/ } / \text {-catenin } \\
\text { mutation/activation }(-) \\
(n=17)\end{array}$ & HR ( $95 \% \mathrm{CI}), p$ values \\
\hline CR, $n(\%)$ & $1(3.7)$ & $0(0)$ & $1(6)$ & $p=0.009$ \\
\hline PR, $n(\%)$ & $2(7.4)$ & $0(0)$ & $2(12)$ & \\
\hline $\mathrm{SD}^{1}, n(\%)$ & $10(37)$ & $0(0)$ & $9(53)$ & \\
\hline PD, $n(\%)$ & $14(51.9)$ & $10(100)$ & $5(29)$ & \\
\hline ORR, $n(\%)$ & $3(11)$ & $0(0)$ & $3(18)$ & \\
\hline DCR, $n(\%)$ & $13(48)$ & $0(0)$ & $12(71)$ & \\
\hline PFS, months & 5.4 & 2.0 & 7.4 & HR $9.2(2.9-2.89), p<0.0001$ \\
\hline OS (months) & 12.9 & 9.1 & 15.2 & HR $2.6(0.76-8.7), p=0.11$ \\
\hline
\end{tabular}

CI, confidence interval; CR, complete response; DCR, disease control rate; HCC, hepatocellular carcinoma; HR, hazard ratio; ICIs, immune checkpoint inhibitors; ORR, objective response rate; OS, overall survival; PD, progressive disease; PFS, progression-free survival; PR, partial response; SD, stable disease. ${ }^{1}$ Durable ( $\geq 4$ months).

patients $(10 / 10)$ with WNT/ $\beta$-catenin mutations showed PD, whereas in the 17 patients without mutations, ICI therapy resulted in a disease control rate of 71\% [16] (Table 2). In addition, progression-free survival after ICI monotherapy was just 2.0 months in patients with WNT/ $\beta$-catenin mutations versus 7.4 months in patients without mutations $(p<0.0001)$ (Table 3). This $71 \%$ figure almost perfectly corresponds to the $70 \%$ figure obtained by 
Table 3. Progression-free survival in patients with advanced HCC treated with ICI or sorafenib [cited from 16]

\begin{tabular}{lllc}
\hline & $\begin{array}{l}\text { WNT/ } \beta \text {-catenin mutation/ } \\
\text { activation }(+)\end{array}$ & $\begin{array}{l}\text { WNT/ } \beta \text {-catenin mutation/ } \\
\text { activation }(-)\end{array}$ & $p$ value \\
\hline ICI $(n=27)$ & 2.0 & 7.4 & $<0.0001$ \\
Sorafenib $(n=79)$ & 4.5 & 5.1 & 0.44 \\
\hline
\end{tabular}

HCC, hepatocellular carcinoma; ICI, immune checkpoint inhibitor, including anti-CTLA-4 monotherapy $(n=1)$, anti-PD-1/PD-L1 monotherapy $(n=25)$, and anti-PD-1/PD-L1 + other ICI (anti-CTLA-4 [ $n=1]$, antiLAG3 [ $n=2]$, and anti-KIR $[n=2])$.

combining the immune hot subclass (30\%) and immune moderate subclass (40\%), as shown in Figure 3. However, WNT/ $\beta$-catenin mutations do not affect the response to sorafenib [16] (Table 3).

WNT $/ \beta$-catenin mutations are detected in $20-30 \%$ of all patients with HCC. PD rates after treatment with nivolumab alone and pembrolizumab alone are high $(37$ and 32.4\%, respectively) [5, 6]. However, addition of an anti-VEGF antibody or TKI to anti-PD-1/PD-L1 antibody markedly reduces the PD rate, which was $20 \%$ for atezolizumab plus bevacizumab in the phase III IMbrave150 study [7], 7.0\% for pembrolizumab plus lenvatinib in a phase $1 \mathrm{~b}$ study [17], and 8.3\% for nivolumab plus lenvatinib in a phase Ib study [18] (Table 4). Anti-PD-1/ PD-L1 antibody monotherapy is associated with a high PD rate of $32.4-42.0 \%[5,6,19]$ (Table 4). The objective response rate of bevacizumab is $13 \%$ by RECIST 1.1 [20], whereas that of lenvatinib is $18.8 \%$ by RECIST 1.1 and $40.6 \%$ by mRECIST [21], indicating a more potent direct antitumor effect of lenvatinib [22] (Table 5). One possible reason for the low PD rate of combination therapy with TKI is that lenvatinib and other multikinase inhibitors cause greater tumor necrosis and release of tumor antigens than bevacizumab, and therefore combination therapy with TKI enhances immune response against Teng's type II HCC. Combination regimens with an ICI plus an anti-VEGF antibody or TKI should be effective against tumors with WNT/ $\beta$-catenin mutations [23-26] (Fig. 3). A low PD rate results in a longer progression-free survival (Table 4).

In summary, the anti-tumor effect of combination immunotherapy is stronger than that of ICI monotherapy due to synergistic effects mediated by multiple factors: (1) anti-VEGF effects (reversal of the immunosuppressive TME, activation of dendritic cells, and increased infiltration of immune cells into tumor); (2) direct antitumor effects (increased release of tumor antigens by blocking the VEGF pathway and multiple kinase pathways); (3) blocking of the PD-1/PD-L1 pathway (activation of CD8-positive cells); and (4) effects in patients with $\mathrm{WNT} / \beta$-catenin mutations.

\section{Conclusion}

Clinical trials of combination therapy with anti-PD-1/PD-L1 antibody and an anti-VEGF antibody or TKI discussed in this editorial are in progress for all stages of HCC (Fig. 7). Combination therapy with an anti-PD-1/PD-L1 antibody and an anti-CTLA-4 antibody [27] is not discussed in this editorial but is currently being investigated in phase III clinical trials. If the effects of anti-PD-1/PD-L1 plus anti-VEGF antibodies reported in the phase III IMbrave150 study are confirmed in future clinical studies of early and intermediate stages of HCC, this therapy should greatly extend patient survival. ICI monotherapy regimens that inhibit only 

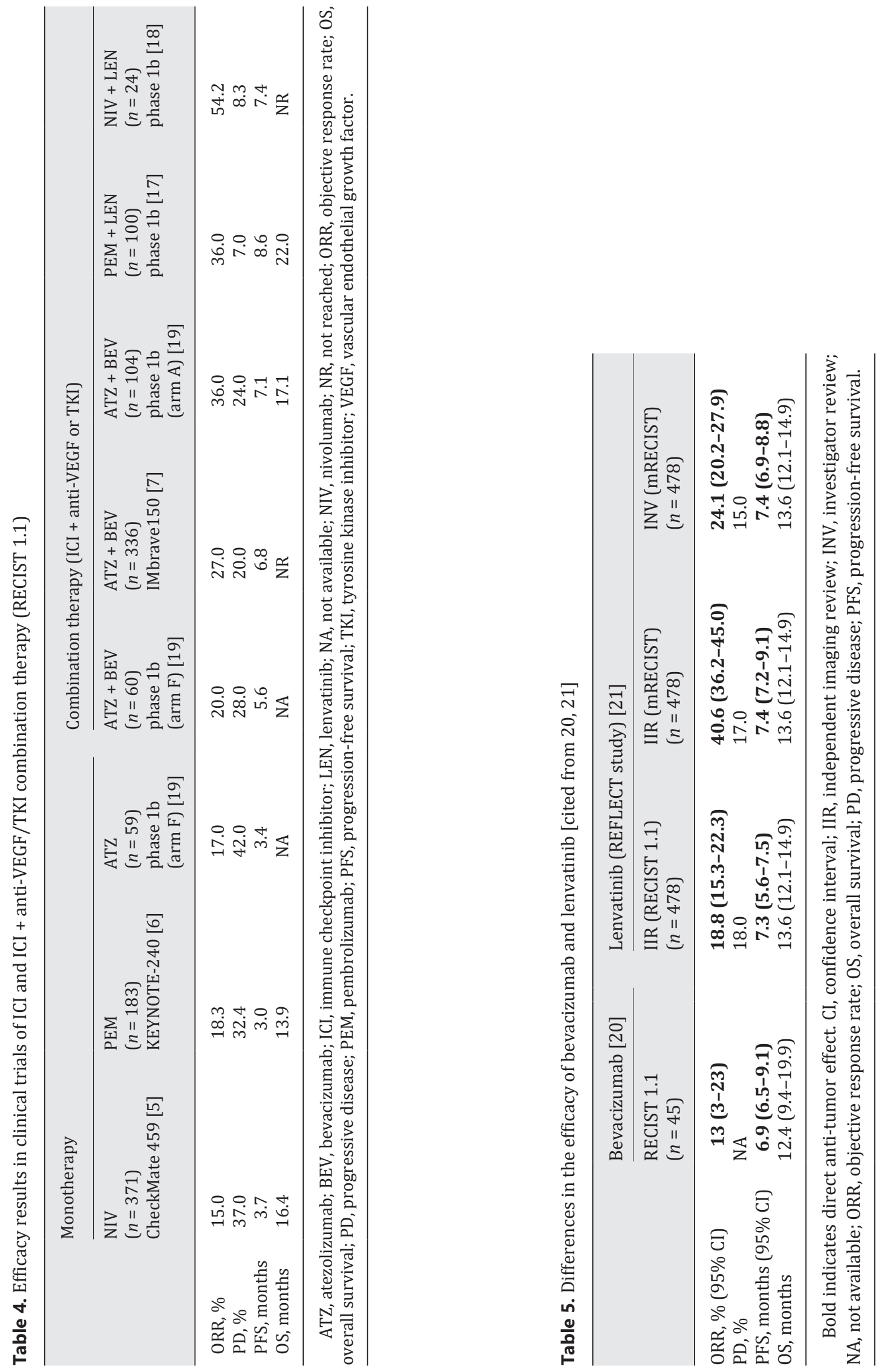


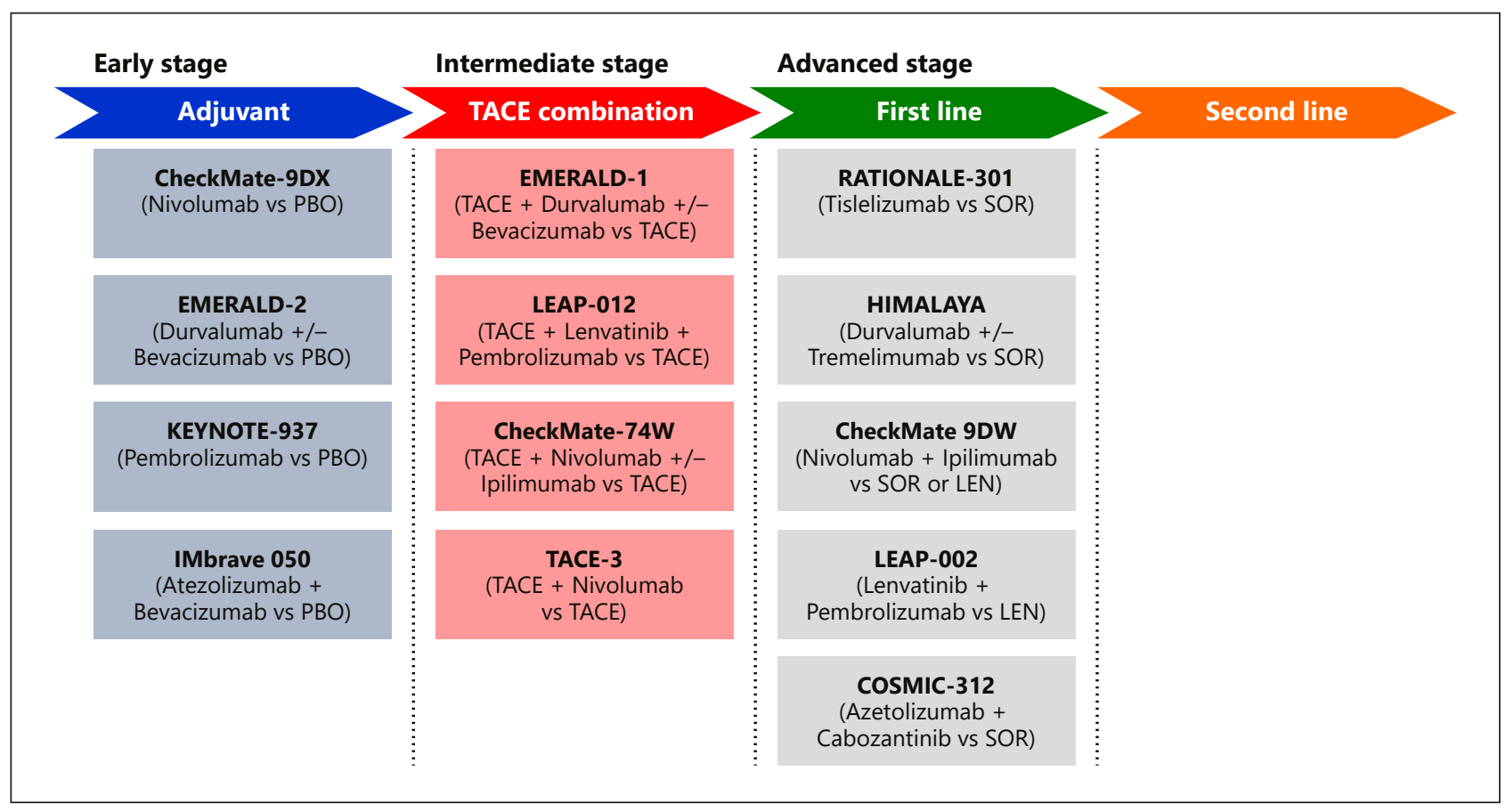

Fig. 7. Ongoing phase III trials in hepatocellular carcinoma.

the PD-1/PD-L1 pathway have a limited impact because they do not produce the synergistic inhibitory effects on VEGF-A or WNT/ $\beta$-catenin mutation that are obtained with combination therapy using an ICI plus an anti-VEGF antibody or TKIs.

\section{Conflict of Interest Statement}

Lectures: Eisai, Bayer, MSD. Grants: EA Pharma, Eisai, Gilead, Takeda, Otsuka, Taiho. Advisory consulting: Eisai, Ono, MSD, BMS.

\section{Funding Sources}

None.

\section{References}

1 Kudo M. Combination Cancer Immunotherapy with Molecular Targeted Agents/Anti-CTLA-4 Antibody for Hepatocellular Carcinoma. Liver Cancer. 2019 Feb;8(1):1-11.

2 Kudo M. Combination Cancer Immunotherapy in Hepatocellular Carcinoma. Liver Cancer. 2018 Mar;7(1): 20-7.

3 El-Khoueiry AB, Sangro B, Yau T, Crocenzi TS, Kudo M, Hsu C, et al. Nivolumab in patients with advanced hepatocellular carcinoma (CheckMate 040): an open-label, non-comparative, phase 1/2 dose escalation and expansion trial. Lancet. 2017 Jun;389(10088):2492-502.

4 Zhu AX, Finn RS, Edeline J, Cattan S, Ogasawara S, Palmer D, et al.; KEYNOTE-224 investigators. Pembrolizumab in patients with advanced hepatocellular carcinoma previously treated with sorafenib (KEYNOTE224): a non-randomised, open-label phase 2 trial. Lancet Oncol. 2018 Jul;19(7):940-52.

5 Yau T, Park JW, Finn RS, Cheng AL, Mathurin P, Edeline J, et al. CheckMate 459: a randomized, multi-center phase III study of nivolumab vs sorafenib as first-line treatment in patients with advanced hepatocellular carcinoma. Ann Oncol. 2019;30(Suppl 5):v874-5. 


\begin{tabular}{|c|c|}
\hline Liver Cancer 2020;9:629-6 & \\
\hline DOI: 10.1159/000512170 & $\begin{array}{l}\text { (c) } 2020 \text { The Author(s). Published by S. Karger AG, Basel } \\
\text { www.karger.com/lic }\end{array}$ \\
\hline
\end{tabular}

Kudo: Anti-PD-1/PD-L1 Monotherapy for HCC

6 Finn RS, Ryoo BY, Merle P, Kudo M, Bouattour M, Lim HY, et al.; KEYNOTE-240 investigators. Pembrolizumab As Second-Line Therapy in Patients With Advanced Hepatocellular Carcinoma in KEYNOTE-240:ARandomized, Double-Blind, Phase III Trial. J Clin Oncol. 2020 Jan;38(3):193-202.

7 Finn RS, Qin S, Ikeda M, Galle PR, Ducreux M, Kim TY, et al.; IMbrave150 Investigators. Atezolizumab plus Bevacizumab in Unresectable Hepatocellular Carcinoma. N Engl J Med. 2020 May;382(20):1894-905.

8 Sia D, Jiao Y, Martinez-Quetglas I, Kuchuk O, Villacorta-Martin C, Castro de Moura M, et al. Identification of an Immune-specific Class of Hepatocellular Carcinoma, Based on Molecular Features. Gastroenterology. 2017 Sep;153(3):812-26.

9 Pinyol R, Sia D, Llovet JM. Immune Exclusion-Wnt/CTNNB1 Class Predicts Resistance to Immunotherapies in HCC. Clin Cancer Res. 2019 Apr;25(7):2021-3.

10 Teng MW, Ngiow SF, Ribas A, Smyth MJ. Classifying Cancers Based on T-cell Infiltration and PD-L1. Cancer Res. 2015 Jun;75(11):2139-45.

11 Chen DS, Mellman I. Oncology meets immunology: the cancer-immunity cycle. Immunity. 2013 Jul;39(1):1-10.

12 Voron T, Marcheteau E, Pernot S, Colussi O, Tartour E, Taieb J, et al. Control of the immune response by proangiogenic factors. Front Oncol. 2014 Apr; 4:70.

13 Kudo M. Scientific Rationale for Combined Immunotherapy with PD-1/PD-L1 Antibodies and VEGF Inhibitors in Advanced Hepatocellular Carcinoma. Cancers (Basel). 2020 Apr;12(5):12.

14 Kudo M. Pembrolizumab for the Treatment of Hepatocellular Carcinoma. Liver Cancer. 2019 May;8(3):14354.

15 Kudo M. Immuno-Oncology Therapy for Hepatocellular Carcinoma: Current Status and Ongoing Trials. Liver Cancer. 2019 Jul;8(4):221-38.

16 Harding JJ, Nandakumar S, Armenia J, Khalil DN, Albano M, Ly M, et al. Prospective genotyping of hepatocellular carcinoma: clinical implications of next-generation sequencing for matching patients to targeted and immune therapies. Clin Cancer Res. 2019 Apr;25(7):2116-26.

17 Finn RS, Ikeda M, Zhu AX, Sung MW, Baron AD, Kudo M, et al. Phase Ib Study of Lenvatinib Plus Pembrolizumab in Patients With Unresectable Hepatocellular Carcinoma. J Clin Oncol. 2020 Sep;38(26):2960-70.

18 Kudo M, Ikeda K, Motomura K, Okusaka T, Kato N, Dutcus CE, et al. A Phase 1b study of lenvatinib plus nivolumab in patients with unresectable hepatocellular carcinoma. ASCO-GI, January 23-25, 2020, San Francisco, USA, Abstr \#513.

19 Lee MS, Ryoo BY, Hsu CH, Numata K, Stein S, Verret W, et al.; G030140 investigators. Atezolizumab with or without bevacizumab in unresectable hepatocellular carcinoma (G030140): an open-label, multicentre, phase 1b study. Lancet Oncol. 2020 Jun;21(6):808-20.

20 Siegel AB, Cohen EI, Ocean A, Lehrer D, Goldenberg A, Knox JJ, et al. Phase II trial evaluating the clinical and biologic effects of bevacizumab in unresectable hepatocellular carcinoma. J Clin Oncol. 2008 Jun;26(18): 2992-8.

21 Kudo M, Finn RS, Qin S, Han KH, Ikeda K, Piscaglia F, et al. Lenvatinib versus sorafenib in first-line treatment of patients with unresectable hepatocellular carcinoma: a randomised phase 3 non-inferiority trial. Lancet. 2018 Mar;391(10126):1163-73.

22 Kudo M. Extremely High Objective Response Rate of Lenvatinib: Its Clinical Relevance and Changing the Treatment Paradigm in Hepatocellular Carcinoma. Liver Cancer. 2018 Sep;7(3):215-24.

23 Kurebayashi Y, Ojima H, Tsujikawa H, Kubota N, Maehara J, Abe Y, et al. Landscape of immune microenvironment in hepatocellular carcinoma and its additional impact on histological and molecular classification. Hepatology. 2018 Sep;68(3):1025-41.

24 Ueno A, Masugi Y, Yamazaki K, Kurebayashi Y, Tsujikawa H, Effendi K, et al. Precision pathology analysis of the development and progression of hepatocellular carcinoma: implication for precision diagnosis of hepatocellular carcinoma. Pathol Int. 2020 Mar;70(3):140-54.

25 Nishida N, Sakai K, Morita M, Aoki T, Takita M, Hagiwara S, et al. Association between genetic and immunological background of hepatocellular carcinoma and expression of programmed cell death-1. Liver Cancer. 2020 Aug;9(4):426-39.

26 Kudo M. Gd-EOB-DTPA-MRI could predict WNT/ $\beta$-catenin mutation and resistance to immune checkpoint inhibitor therapy in hepatocellular carcinoma. Liver Cancer. 2020;9(5):479-90.

27 Kudo M. Scientific rationale for combination immunotherapy of hepatocellular carcinoma with anti-PD-1/ PD-L1 and anti-CTLA-4 antibodies. Liver Cancer. 2019 Nov;8(6):413-26. 